\title{
Lab-on-capillary platform for on-site quantitative SERS analysis of surface contaminants based on Au@4-MBA@Ag core-shell
} nanorods

\author{
Shuang Lin, ${ }^{\ddagger}$ Wuliji Hasi, ${ }^{*}+X i a n g$ Lin, ${ }^{\ddagger}$ Siqingaowa Han, ${ }^{\dagger}$ Ting Xiang, ${ }^{\ddagger}$ Shan Liang ${ }^{\ddagger}$, and Li \\ Wang ${ }^{*}$
}

${ }^{\dagger}$ National Key Laboratory of Science and Technology on Tunable Laser, Harbin Institute of Technology, Harbin, 150080, P. R. China.

${ }^{\ddagger}$ School of Physics and Materials Engineering, Dalian Minzu University, Dalian, 116600 P. R. China.

§Affiliated Hospital, Inner Mongolia University for the Nationalities, Tongliao, 028007, P. R. China.

\section{EXPERIMENTAL SECTION}

\section{Materials}

Cetyltrime-thylammonium bromide (CTAB) and polyvinyl pyrrolidone (PVP) were obtained from Sigma-Aldrich Co., Ltd., sodium borohydride $\left(\mathrm{NaBH}_{4}\right)$ and ascorbic acid (AA) were obtained from Acros Organics Co., Ltd., cetyltrime-thylammonium chloride (CTAC, 97\%) and 3-Aminopropyl)-triethoxysilane (APTES, 98\%) were obtained from Aladdin Chemical Co., Ltd., silver nitrate $\left(\mathrm{AgNO}_{3}\right)$ and chloroauric acid $\left(\mathrm{HAuCl}_{4}\right)$ were obtained from Sinopharm Chemical reagent Co. Ltd., malachite green (MG) and crystal violet (CV) were obtained from Adamas Reagent Co., Ltd. All the reagents were used as received. Deionized water with a resistivity of $18.2 \mathrm{M} \Omega \mathrm{cm}^{-1}$ obtained from a Direct-Q 3 UV water purification system was used in all experiments.

Synthesis of Au nanobipyramids (NBPs)

Au NBP sample was prepared using the seed-mediated growth method, as described in previous works. ${ }^{1}$ Firstly, twinned gold seeds were prepared via a thermal treatment procedure. Then a growth solution was made by mixing together CTAB solution (10o mL, $100 \mathrm{mM}), \mathrm{HAuCl}_{4}(2 \mathrm{~mL}, 25 \mathrm{mM}), \mathrm{AgNO}_{3}(1 \mathrm{~mL}, 10 \mathrm{mM}), \mathrm{HCl}(2 \mathrm{~mL}$, $1 \mathrm{M})$, and AA $(0.8 \mathrm{~mL}, 100 \mathrm{mM})$. The seed solution $(0.5 \mathrm{~mL})$ was injected into the growth solution under vigorous stirring. The reaction solution was left undisturbed for 2 hours at room temperature. The longitudinal plasmon wavelength of the obtained Au NBP sample was $\sim 820 \mathrm{~nm}$. The Au NBPs were centrifuged and the precipitate was re-dispersed in water (10 mL).

\section{Synthesis of Au nanorods (NRs)}

Big Au NRs were prepared using the initial Au NBPs as seed according to the previous work. ${ }^{2}$ Growth solutions were made by mixing together $\mathrm{CTAB}$ solution $(40 \mathrm{~mL}, 100 \mathrm{mM}), \mathrm{HAuCl}_{4}(0.4 \mathrm{~mL}, 25 \mathrm{mM}), \mathrm{HCl}(0.8 \mathrm{~mL}, 1 \mathrm{M})$, and AA (o.16 $\mathrm{mL}, 100 \mathrm{mM}$ ) only without $\mathrm{AgNO}_{3}$. The Au NDP solutions of $\mathrm{mLL}$ were employed as seed and injected into the growth solution under vigorous stirring. The reaction solution was left undisturbed for 2 hours at room temperature. The big Au NRs were centrifuged and the precipitate was re-dispersed in water $(4 \mathrm{~mL})$.

\section{Synthesis of Au NRs@Ag NRs}

Au NR solutions ( $1 \mathrm{~mL}$ ) were added into CTAC solution (20 mL, 20 mM) at $65{ }^{\circ} \mathrm{C}$ under magnetic stirring, followed by subsequent addition and mixing of $\mathrm{AgNO}_{3}(75 \mathrm{mM}, 150 \mu \mathrm{L})$ and $\mathrm{AA}(75 \mathrm{mM}, 1.5 \mathrm{~mL})$. The mixture solution was kept for $4.5 \mathrm{~h}$ at $65^{\circ} \mathrm{C}$. The resultant samples were centrifuged and the precipitate was re-dispersed in CTAC solution (20 $\mathrm{mL}, 1 \mathrm{mM}$ ) for further use.

\section{Fabrication of capillary-based substrate}

In brief, CTAC-coated Au@Ag NRs were converted to PVP-coated Au@Ag NRs by twice centrifugation. The specific details process can be referred from our previous work. ${ }^{2}$ Firstly, a mixed solution of acetone and ethanol was used to 
ultrasonically clean capillary for to minutes, followed by rinsed with water and dried. Then the capillary was immersed in the piranha solution $\left(\mathrm{H}_{2} \mathrm{SO}_{4}: \mathrm{H}_{2} \mathrm{O}_{2}=4: 1, \mathrm{~V} / \mathrm{V}\right)$ for $2 \mathrm{~h}$, followed by rinsed with water and dried. Finally, the capillary was immersed in APTES ethanol solution with a volume fraction of $1 \%$ for $12 \mathrm{~h}$, followed by rinsed with ethanol and dried. ${ }^{3}$ The treated capillaries were modified with positively charged amino groups to better adsorb negatively charged PVP-coated nanoparticles. Specifically, the treated capillary was immersed vertically in a concentrated Au@Ag NR colloid. The immersing time was adjusted from $1 \mathrm{~h}$ to $24 \mathrm{~h}$, and the concentration times of the nanoparticles was adjusted from 5 to 100 times. Similarly, Si wafers and quartz slides were also functionalized with APTES as same as capillary, and then immersed in colloids as a comparative examination.

\section{Preparation of $\mathrm{CV}$ and MG spiked shell samples}

To prepare CV standard solution, $0.0407 \mathrm{~g} \mathrm{CV}$ powder was weighed and then dissolved it into $100 \mathrm{~mL}$ water. Aqueous solutions of CV with gradient concentrations were prepared by diluting CV standard solution with different proportions. Similarly, to prepare MG standard solution, $0.0927 \mathrm{~g}$ MG powder was weighed and then dissolved it into $100 \mathrm{~mL}$ water. Aqueous solutions of MG with gradient concentrations were prepared by diluting MG standard solution with different proportions. To prepare spiked shell samples, the shells obtained from local market were firstly washed cleanly and then immersed in CV aqueous solution and MG aqueous solution for $2 \mathrm{~h}$, respectively, followed by dried. Then a small amount of ethanol was spared on the surface of shell. Finally, the capillary-based substrate was touched on the shell to extra the samples for detection.

\section{Characterization and SERS measurement}

UV-visible spectra were acquired with a Lambda 750 UV/Vis/NIR spectrophotometer. Spectral analysis was performed in the range of $300 \sim 2000 \mathrm{~nm}$ at room temperature using quartz cuvettes with optical path length of $1 \mathrm{~cm}$. Scanning electron microscopy (SEM) images were carried out using a microscopy (model S-48oo, Hitachi, Japan) with an acceleration voltage of $5.0 \mathrm{kV}$. Transmission electron microscope (TEM) images were taken by a JEM-210o TEM instrument (JEOL, Japan).

Raman spectra were recorded using a portable compact laser Raman Spectrometer (BWS415-785H, B\&W Tek, Inc.) at the excitation wavelength of $785 \mathrm{~nm}$ and the total laser energy is $300 \mathrm{~mW}$. The spectrum range of the spectrometer is from 175 to $2700 \mathrm{~cm}^{-1}$ and the spectral resolution is better than $3.5 \mathrm{~cm}^{-1}$.

\section{AEF calculation}

In detail, the normal Raman spectra of $10^{-1} \mathrm{M}$ R6G aqueous solution using capillary substrate without loading $\mathrm{Au} @ \mathrm{Ag}$ nanorods were recorded (Fig. S5). AEF was calculated according to the following equation: ${ }^{4}$

$$
A E F=\frac{I_{\text {SERS }}}{I_{\text {normal }}} \times \frac{C_{\text {normal }}}{C_{S E R S}}
$$

where $I_{S E R S}$ is the intensity of the band at $611 \mathrm{~cm}^{-1}$ obtained by SERS, $I_{\text {normal }}$ is the corresponding band intensity of the normal Raman spectra. $C_{\text {SERS }}$ and $C_{\text {normal }}$ are the concentrations in the SERS and normal Raman measurements, respectively. 

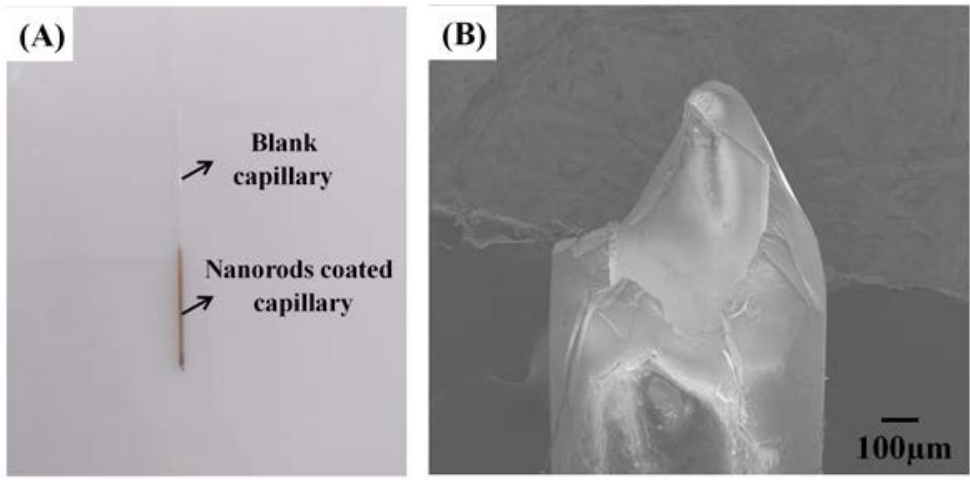

Figure S1. (A) The photograph of blank capillary and nanorods coated capillary; (B) SEM image of a broken section of the capillary tube.
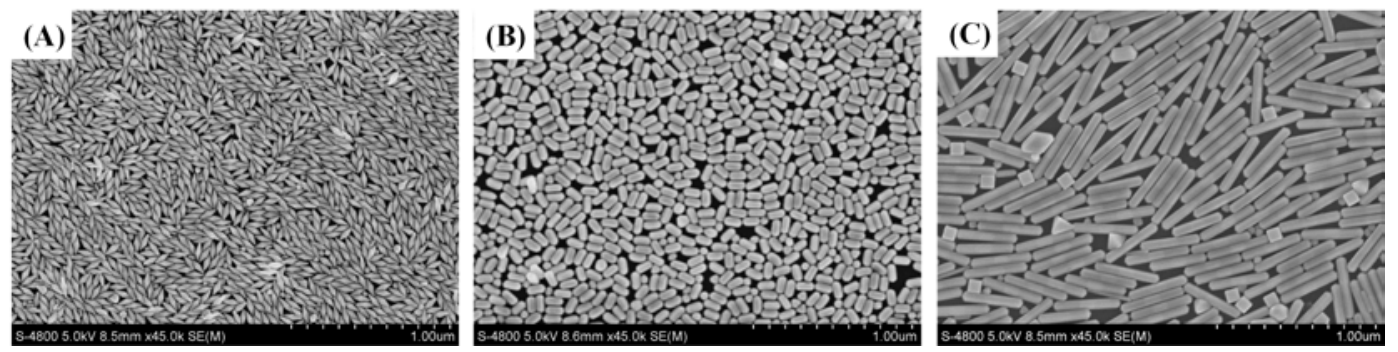

Figure S2. (A) SEM image of Au NBPs; (B) SEM image of Au NRs by the overgrowth of Au NBPs; (C) SEM image of Au NRs@Ag NRs.
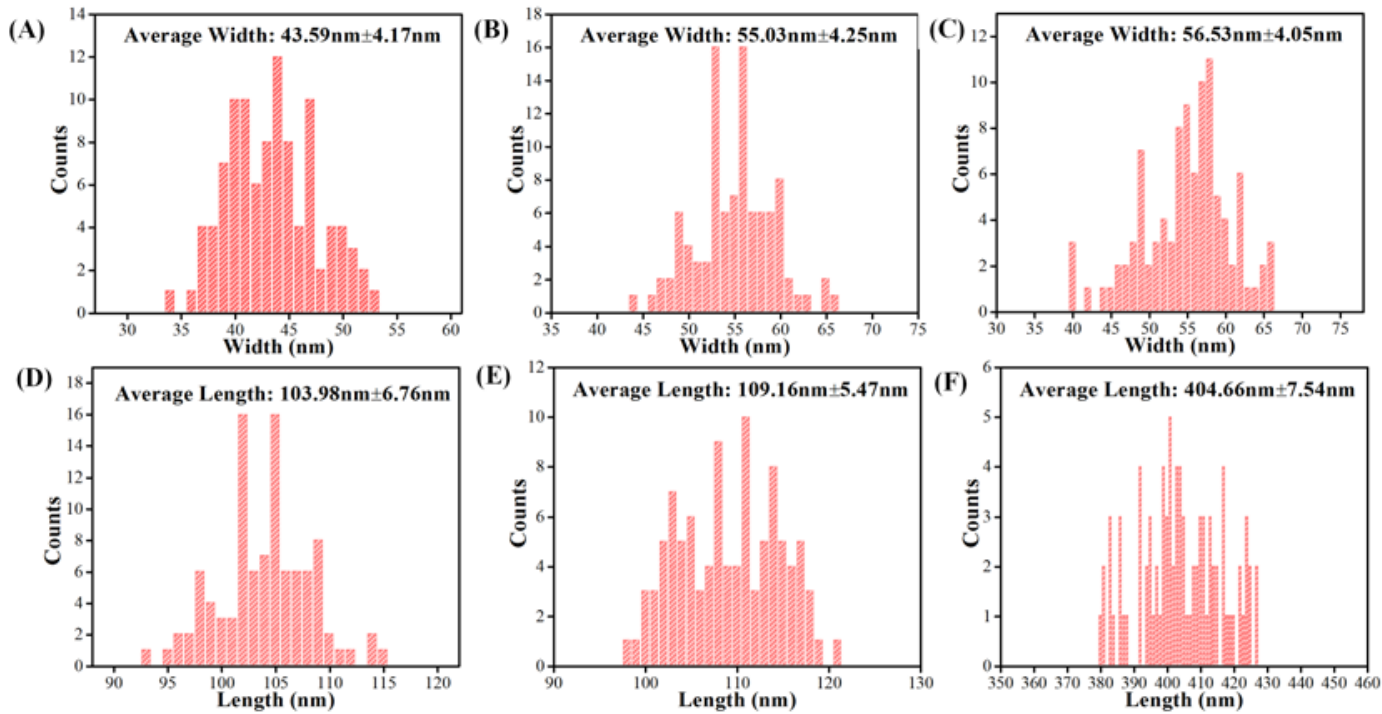

Figure S3. The corresponding particle size distribution of Au NBPs (A) and (D); Au NRs (B) and (E); Au NRs@Ag NRs (C) and (F).
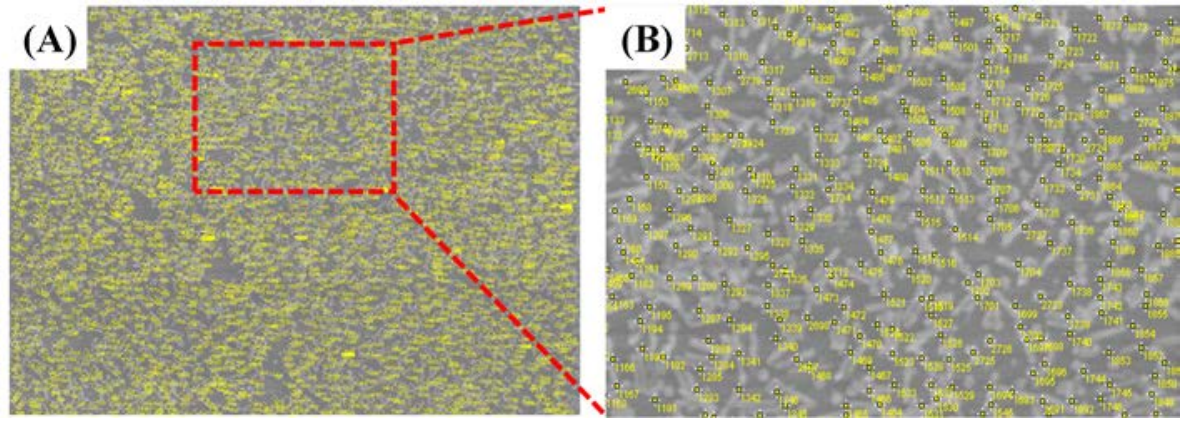

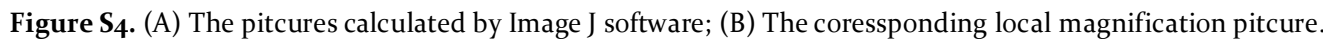




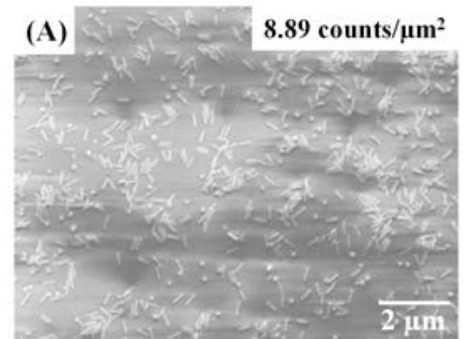

(D)

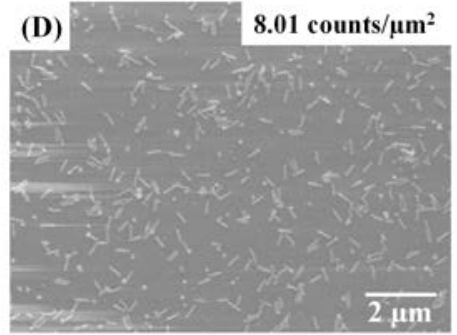

(B)

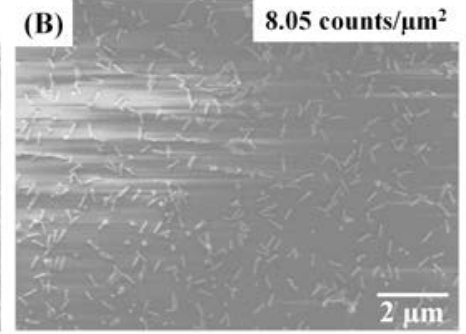

(E)

8.24 counts $/ \mu \mathrm{m}^{2}$

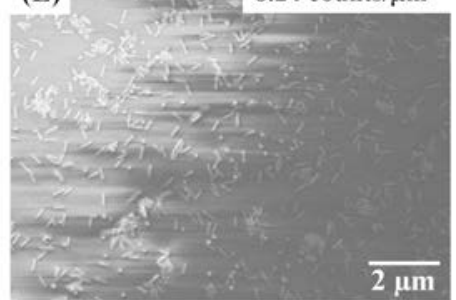

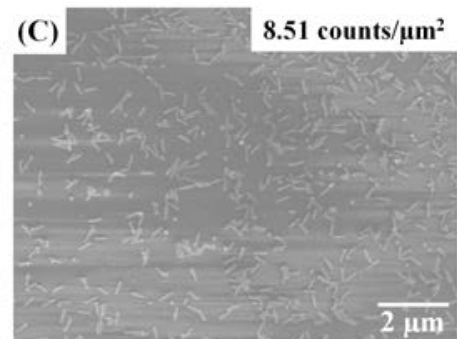

(F)
8.33 counts $/ \mu \mathrm{m}^{2}$

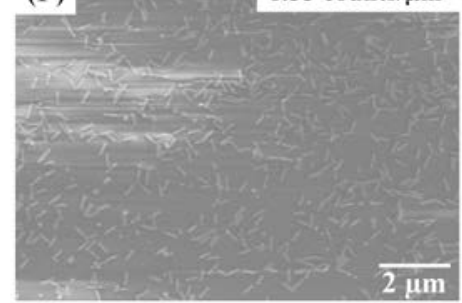

Figure S5. (A) (F) SEM images of 6 ramdon positions on the same broken capillary-based substrate decorated with Au@Ag NRs concentrated by 10 time immersing in colloids.

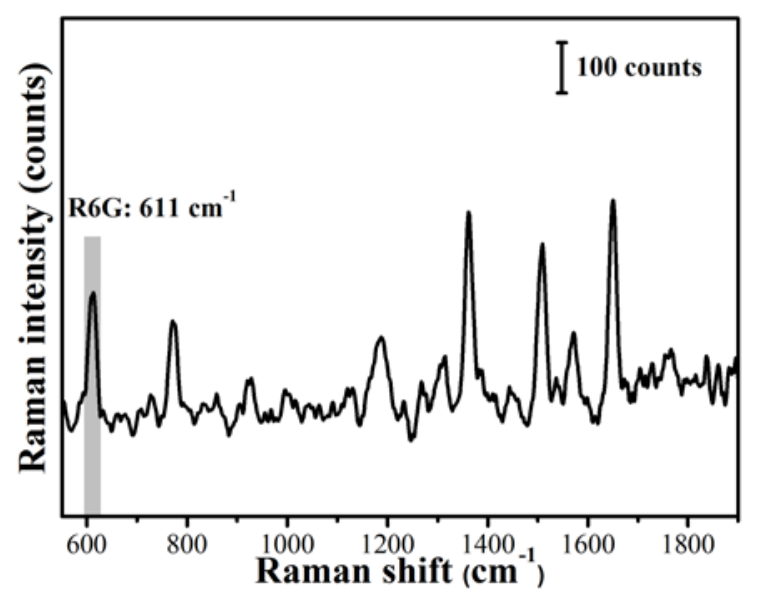

Figure S6. Normal Raman spectrum of R6G molecule at the concentration of $10^{-1} \mathrm{M}$. The excitation wavelength is $785 \mathrm{~nm}$, the laser power is $50 \%$ and the integration time is $10 \mathrm{~s}$ for all experiments. 

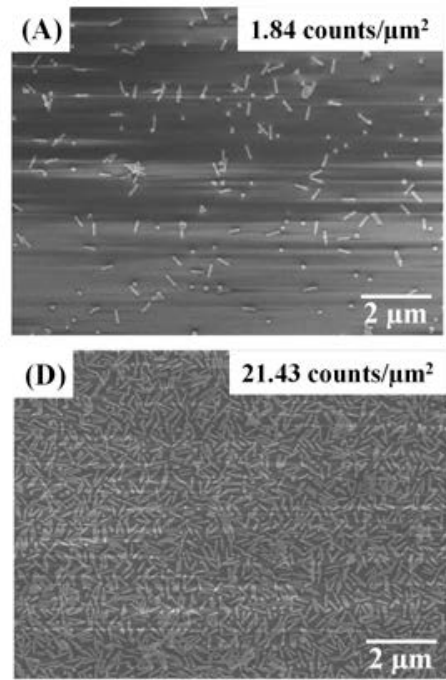

(G)

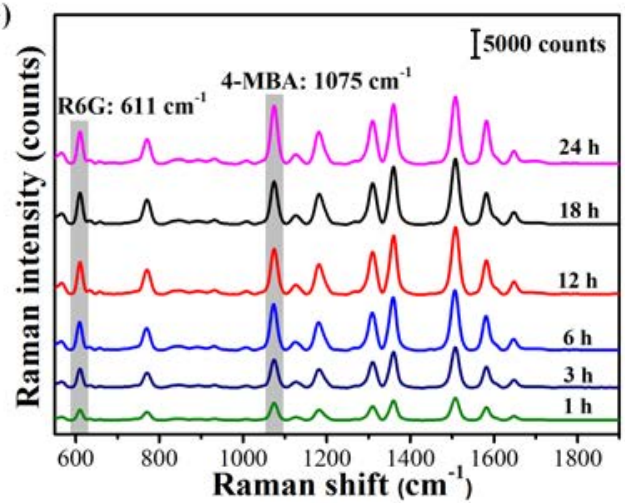

(E)
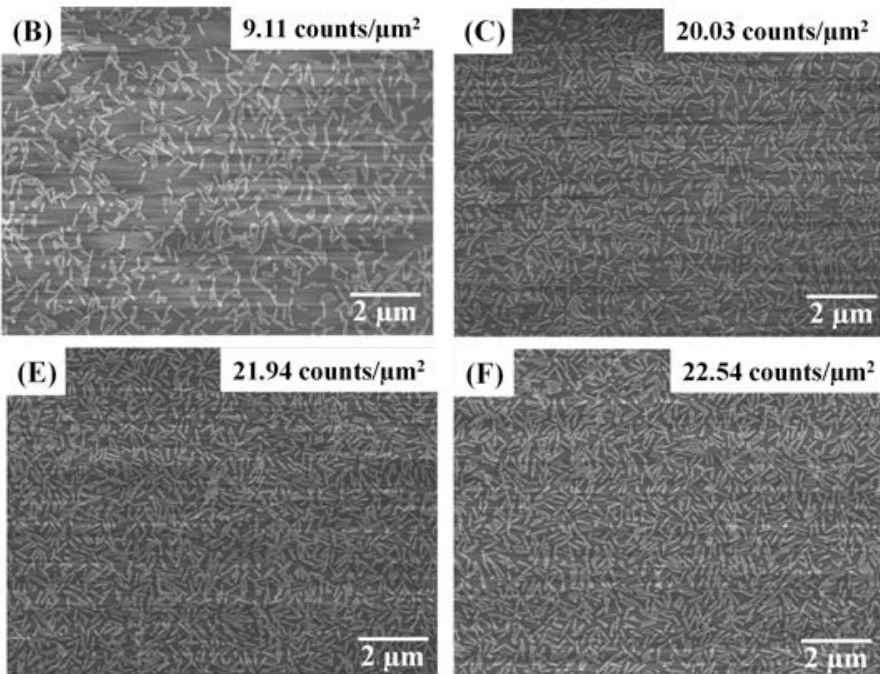

(F)

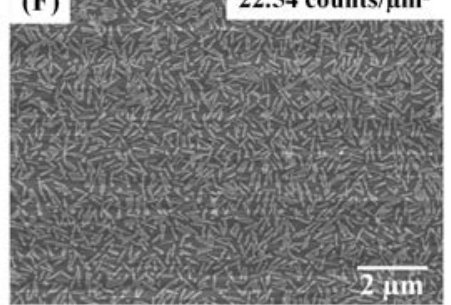

(H)

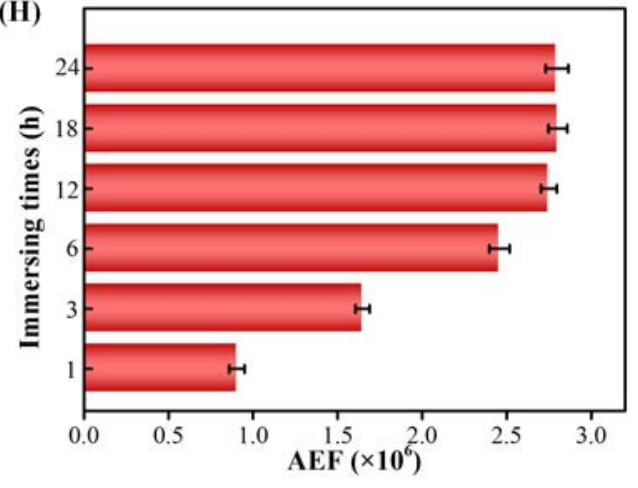

Figure S7. (A) (F) SEM images of capillary-based substrate decorated with Au@Ag NRs concentrated by 50 time immersing in colloids for 1 h, $3 \mathrm{~h}, 6 \mathrm{~h}, 12 \mathrm{~h}, 18 \mathrm{~h}$ and $24 \mathrm{~h}$, respectively; (G) SERS spectra of R6G $\left(10^{-5} \mathrm{M}\right)$ based on capillary-based substrate decorated with Au@Ag NRs concentrated by 50 time immersing in colloids for $1 \mathrm{~h}, 3 \mathrm{~h}, 6 \mathrm{~h}, 12 \mathrm{~h}, 18 \mathrm{~h}$ and $24 \mathrm{~h}$; $(\mathrm{H})$ The corresponding histogram for the AEF results of probe molecule R6G $\left(61 \mathrm{~cm}^{-1}\right)$. The excitation wavelength is $785 \mathrm{~nm}$, the laser power is $50 \%$ and the integration time is $10 \mathrm{~s}$ for all experiments. Each spectrum represents the average value from five SERS spectra, error bars were obtained from five measurements at each concentration.
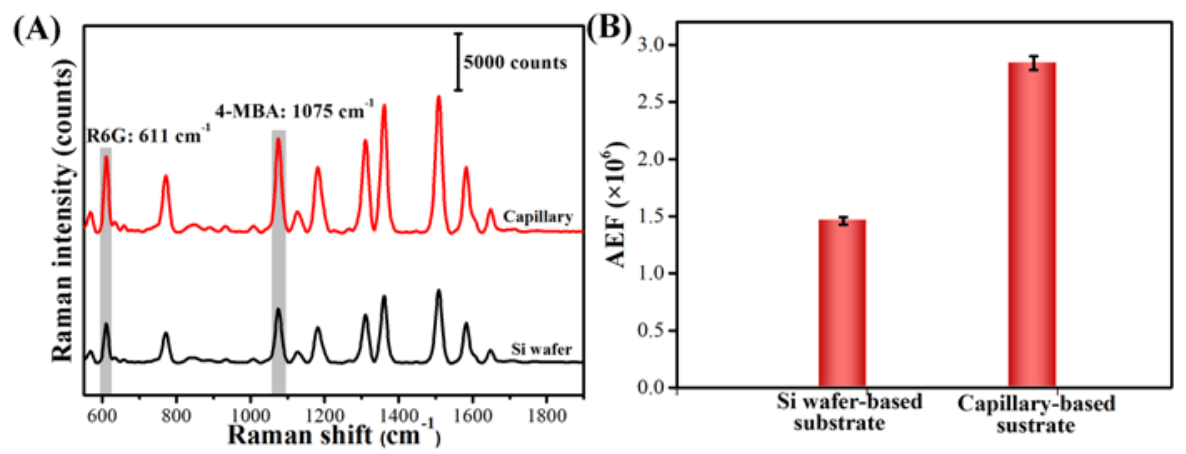

Figure S8. (A) SERS spectra of R6G $\left(10^{-5} \mathrm{M}\right)$ based on Si wafer-based substrate and capillary-based substrate decorated with Au@Ag NRs concentrated by 50 time immersing in colloids for $12 \mathrm{~h}$. (B) The corresponding histogram for the AEF results of probe molecule R6G $\left(611 \mathrm{~cm}^{-1}\right)$. The excitation wavelength is $785 \mathrm{~nm}$, the laser power is $50 \%$ and the integration time is $10 \mathrm{~s}$ for all experiments. Each spectrum represents the average value from five SERS spectra, error bars were obtained from five measurements at each concentration.

\section{REFERENCES}

(1). Lee, J. H.; Gibson, K. J.; Chen, G.; Weizmann, Y. Bipyramid-templated synthesis of monodisperse anisotropic gold nanocrystals.

Nat. Commun. 2015, 6, 7571. 
(2). Lin, S.; Lin, X.; Han, S.; He, L.; Zhao, H.; Zhang, J.; Hasi, W.; Wang, L. Width and length dependent SERS performance of core-shell Au@ Ag nanorod self-assembled monolayers. J. Alloy Compound 2019, 805, 318-326.

(3). Yüksel, S.; Schwenke, A. M.; Soliveri, G.; Ardizzone, S.; Weber, K.; Cialla-May, D.; Hoeppener, S.; Schubert, U. S.; Popp, J. Trace detection of tetrahydrocannabinol (THC) with a SERS-based capillary platform prepared by the in situ microwave synthesis of AgNPs. Anal. Chim. Acta 2016, 939, 93-100.

(4) Lin, S.; Lin, X.; Liu, Y.; Zhao, H.; Hasi, W.; Wang, L. Self-assembly of Au@ Ag core-shell nanocubes embedded with an internal standard for reliable quantitative SERS measurements. Anal. Methods 2018, 10(34), 4201-4208. 